\title{
Overexpression of the Rosa rugosa RrGT1 Gene Induces Anthocyanin Accumulation in Tobacco
}

\author{
Xiaoming Sui*, Mingyuan Zhao*, Xu Han, Lanyong Zhao\#, Zongda Xu\# \\ College of Forestry, Shandong Agricultural University, Taian, China \\ Correspondence to: Lanyong Zhao, "sdzly369@163.com; Zongda Xu, "xuzoda@163.com \\ Keywords: Rosa rugosa, RrGT1 Gene, Gene Expression, Overexpression, Tobacco, Anthocyanin \\ Received: September 3, $2018 \quad$ Accepted: October 13, $2018 \quad$ Published: October 16, 2018 \\ Copyright $\odot 2018$ by authors and Scientific Research Publishing Inc. \\ This work is licensed under the Creative Commons Attribution International License (CC BY 4.0). \\ http://creativecommons.org/licenses/by/4.0/

\section{(c) (1) Open Access}

\section{ABSTRACT}

Rosa rugosa has always been an important plant in landscape application, and the improvements and innovations about its flower color are particularly important. Glycosylation modification fulfills an important role in increasing the stability and solubility of anthocyanin in plants. In this study, based on the transcriptional database of $R$. rugosa, a gene with full length cDNA of $1161 \mathrm{bp}$, encoding 386 amino acids, designated as $R r G T 1$, were isolated from flowers of $R$. rugosa "Zizhi" and then functionally characterized. Sequence alignments with the NCBI database show that the $R r G T 1$ protein is a member of the GTB superfamily and has typical conserved amino acid residues called PSPG that are crucial for RrGT1 enzyme activity. $R r G T 1$ transcripts were detected in five flowering stages and seven tissues of $R$. rugosa "Zizhi" and their expression patterns corresponded with the accumulation of anthocyanins. Additionally, the in vivo function of $R r G T 1$ was investigated via its overexpression in tobacco. Transgenic tobacco plants expressing $R r G T 1$ induced anthocyanin accumulation in flowers, indicating that $\operatorname{RrGT1}$ could encode a functional glycosyltransferase (GT) protein for anthocyanin biosynthesis and could function in other species. Therefore, we speculated that glycosylation of $R r G T 1$ played a crucial role in anthocyanin biosynthesis in $R$. rugosa.

\section{INTRODUCTION}

Rosa rugosa is an important ornamental plant which belongs to the genus Rosa in the family Rosaceae. It is native to China and is widely distributed in the world. Because of its unique fragrance, color, cold resistance and drought resistance, it has great development potential in garden application [1]. There are many varieties of roses, but most of them are traditional colors such as pink, purple, etc. A few varieties are white, lacking yellow, bright red, orange and compound color, etc. [2]. Therefore, how to innovate 
rose color has become the main goal of breeders. The analysis of the pigment composition of rose and the study of the expression characteristics of the key enzymes encoding genes that catalyze the synthesis of rose pigment are the important prerequisite for rose color oriented molecular breeding [3]. Anthocyanin determines the color of higher plant organs. Its biosynthesis pathway related structural genes ( $\mathrm{CHS}, \mathrm{CHI}$, $F 3 H, F 3^{\prime} H, D F R, A N S, 3 G T$ etc.) and regulatory genes ( $M Y B$, mostly $R 2 R 3-M Y B, B H L H$ and $W D 40$ classes) have been cloned, sequenced and protein function studies in many plants, such as petunia, maize, snapdragon and so on [4-13]. But less research has been done on $R$. rugosa.

Anthocyanins, derived from the anthocyanin biosynthesis pathway, are the largest group of water-soluble plant flavonoids found in organs of plants and crops [14] [15]. Anthocyanins are unstable in plants, mainly in the form of glycosides in the vacuole [16]. Anthocyanins play an important role in insect pollination, auxin transport, protection of leaves from ultraviolet radiation, inhibition of diseases and insect pests, etc. [17]. In addition, as a safe, non-toxic natural food pigment, anthocyanins also have anti-oxidation, anti-cancer and anti-arteriosclerosis functions [7].

Anthocyanin biosynthesis pathway is one of the secondary metabolic pathways in plants. At present, the research on it has been more clear. Firstly, naringin was formed by the catalysis of $C H S$ and $C H I$ with coumaryl coenzyme A and malonyl Co A. Then flavonols were formed under the action of $F 3 H$. The next step is to form colored anthocyanins under the action of DFR and $A N S$. Finally, through glycosylation, methylation, acylation, hydroxylation and other modifications to form a variety of anthocyanins with a stable structure [18]. Flavonoid 3-0-glycosyltransferase (3GT) gene is a downstream gene in anthocyanin synthesis pathway. It can catalyze the glycosylation of UDP glucose to replace the 3 hydroxyl groups of anthocyanin and make anthocyanin glycosylation to produce colored and stable anthocyanins. And move the maximum absorption spectrum to the ultraviolet end, thus increasing the blue tone of anthocyanins [19]. Glycosylation can change the hydrophilicity, biochemical activity and subcellular localization of anthocyanins, which is beneficial to the transport and storage of anthocyanins in cells and organisms [20].

Some studies have shown that the anthocyanin content of plants lacking $3 G T$ also decreased significantly [21]. 3GT gene belongs to a glycosyltransferase (GTs) family 1, whose enzyme protein has a conserved domain of about 44 amino acids at its C-terminal, known as plant secondary product glycosyltransferase (PSPG) box. At present, $3 G T$ has been cloned and analyzed in many plants such as Zea mays [22], Vitis vinifera [23], Gentiana trflora [24], Petunia hybrida [25] and so on, which has laid a foundation for understanding the metabolic regulation of anthocyanin synthesis pathway.

At present, the studies on $R$. rugosa are mainly focused on morphological classification, geographical distribution, essential oil extraction and food quality evaluation, and there are few reports on the anthocyanin biosynthesis mechanism, so we don't know exactly how it works. In this study, based on the $R$. rugosa transcriptome data, we cloned and identified $R r G T 1$ gene from the petals of $R$. rugosa "Zizhi". Stable transformation of the $R r G T 1$ gene in tobacco showed that its overexpression was positively correlated with the accumulation of anthocyanins. We hope that the results of our research can provide some useful informations for the subsequent color improvement project in $R$. rugosa.

\section{MATERIALS AND METHODS}

\subsection{Plant Materials}

For $R$. rugosa, $R$. rugosa "Zizhi" plants cultivated in the Rosa germplasm nursery of Shandong Agricultural University were used as test material. We collected petals at the budding stage, initial opening stage, half opening stage, full opening stage and wilting stage as well as seven different tissue samples (roots, stems, leaves, petals at the budding stage, sepals, stamens and pistils) in the mornings of sunny days from 20 April to 10 May 2017. After flash freezing in liquid nitrogen, all samples, which were collected in triplicate, were put into a $-80^{\circ} \mathrm{C}$ refrigerator for storage.

For tobacco, the wild type was used as transgenic material. After the disinfection of soaking tobacco seeds with $70 \%-75 \%$ ethanol for 2 min, rinsing them with aseptic water once, soaking with $3.5 \% \mathrm{NaClO}$ for $10-15 \mathrm{~min}$, then rinsing with aseptic water for 5 times, the seeds were sown in Murashige and Skoog 
(MS) solid medium (without antibiotics). After 3 days of vernalization at $4^{\circ} \mathrm{C}$ in darkness, the seeds were placed in a growth chamber $\left(25^{\circ} \mathrm{C}, 16 \mathrm{~h} / 23^{\circ} \mathrm{C}, 8 \mathrm{~h}\right.$ day/night, $60 \%$ relative humidity) for approximately 30 days. The germless tobacco seedlings with good growth conditions were selected as follow-up experimental materials.

\subsection{Extraction of Total RNA and Synthesis of First-Strand cDNA}

The total RNA was extracted via an EASY Spin Plant RNA Rapid Extraction Kit (Aidlab Biotech, Beijing, China) in accordance with the manufacturer's specifications. The integrity of the RNA was measured by gel electrophoresis with $1.0 \%$ nondenatured agarose (Figure 1), the purity and concentration of the RNA were detected by a Nanodrop $2000 \mathrm{C}$ ultra-microspectrophotometer (Thermo Fisher Scientific, Wilmington, Delaware, USA), and the qualified RNA was preserved at $-80^{\circ} \mathrm{C}$. First-strand cDNA was synthesized via a $5 \times$ All-In-One RT MasterMix Reverse Transcription Kit (ABM Company, Vancouver, Canada) in accordance with both the manufacturer's protocol and the requirements of RT-PCR and qRT-PCR.

\subsection{Full-Length CDS Cloning of the RrGT1 Gene}

3'RACE specific primers were designed based on the sequence information provided by the $R$. rugosa transcriptome data. The cDNA 3'terminal sequence of the target gene was amplified by 3'RACE technology. The known target gene sequence in transcriptome data and the 3'terminal sequence obtained by RACE technique were spliced with DNAMAN software to obtain the full-length cDNA sequence of the $R r G T 1$ gene. According to the sequence obtained by splicing, the upstream primer RrGT1-F containing the starting codon of the RrGT1 gene and the downstream primer RrGT1-R containing the terminating codon were designed and amplified. The estimated length of amplification was $1161 \mathrm{bp}$.

\subsection{Tobacco Stable Transformation}

The plasmids of a pCAMBIA1304 vector and RrGT1 gene with restriction sites (SpeI and BstEII) were extracted and digested by double enzymes. The digestion products were then ligated with DNA ligase and transformed into Agrobacterium tumefaciens.

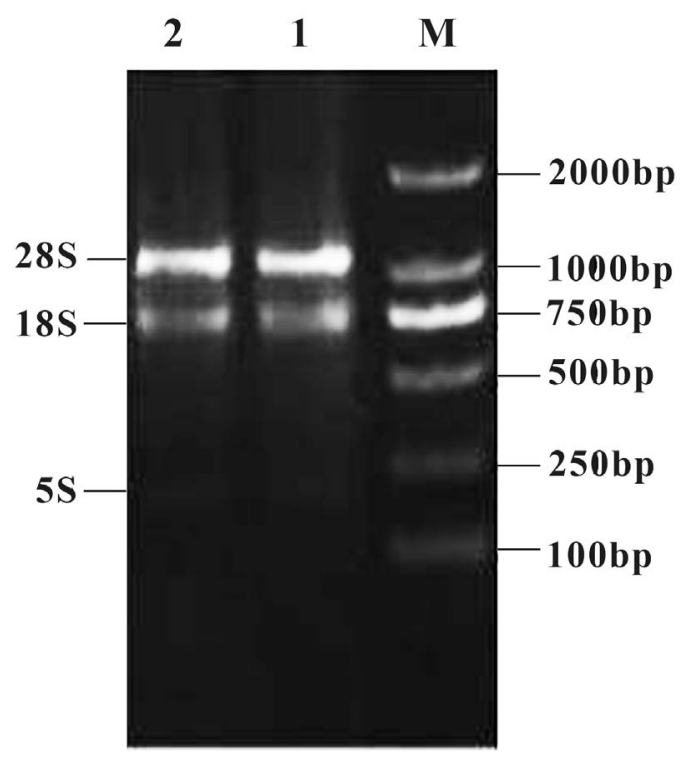

Figure 1. The total RNA electrophoresis diagram. M, Maker; 1and 2, RNA bands. 
A. tumefaciens mediated leaf disks transformation [26] was used to transform tobacco. Firstly, the leaves of the cultured tobacco sterile seedlings were pruned to the appropriate size. A. tumefaciens infection was carried out after 2 days of pre culture. Acetosyringone (AS) was added to the infective liquid, and the pCAMBIA1304 empty carrier was used as the control. After infection, the plants were subjected to a dark treatment for 2 days, after which they were allowed to be cultured in a growth chamber $\left(25^{\circ} \mathrm{C}, 16\right.$ $\mathrm{h} / 23^{\circ} \mathrm{C}, 8 \mathrm{~h}$ day/night, $60 \%$ relative humidity). Differentiation culture and rooting culture were carried out on MS medium supplemented with related hormones and antibiotics. Hygromycin (Hyg) was used to screen resistant seedlings. The plantlets with good growth potential were transplanted into small flowerpots with substrate after seedling refining, and the original growth environment was maintained.

\section{5. qRT-PCR Detection}

We analyzed the gene expression by qRT-PCR on a Bio-Rad CFX96 ${ }^{\mathrm{TM}}$ Real-Time PCR instrument (Bio-Rad, Inc., USA). The qRT-PCR mixture (total volume of $20 \mu \mathrm{L}$ ) contained $10 \mu \mathrm{L}$ of SYBR ${ }^{\circledR}$ Premix Ex $\mathrm{Taq}^{\mathrm{TM}}$ (TaKaRa, Inc., Japan), $8.2 \mu \mathrm{L}$ of $\mathrm{ddH}_{2} \mathrm{O}, 0.4 \mu \mathrm{L}$ of each primer and $1 \mu \mathrm{L}$ of cDNA. The PCR program consisted of an initial step of $95^{\circ} \mathrm{C}$ for $30 \mathrm{~s} ; 40$ cycles of $95^{\circ} \mathrm{C}$ for $5 \mathrm{~s}$ and $60^{\circ} \mathrm{C}$ for $30 \mathrm{~s}$; and then a dissociation stage of $95^{\circ} \mathrm{C}$ for $10 \mathrm{~s}, 65^{\circ} \mathrm{C}$ for $5 \mathrm{~s}$ and $95^{\circ} \mathrm{C}$ for $5 \mathrm{~s}$. Each gene was assessed via three biological replicates. The relative expression levels of the genes were calculated by the $2^{-\Delta \Delta \mathrm{Ct}}$ method [27].

\subsection{Total Anthocyanin Extractions and HPLC Analysis}

All samples ( $0.1 \mathrm{~g}$ fresh weight) were homogenized in liquid nitrogen, after which they were extracted with $5 \mathrm{~mL}$ of an acidic methanol solution (70:0.1:29.9, v/v/v; $\left.\mathrm{CH}_{3} \mathrm{OH}: \mathrm{HCl}: \mathrm{H}_{2} \mathrm{O}\right)$ at $4^{\circ} \mathrm{C}$ in darkness for $24 \mathrm{~h}$ and then sonicated for $30 \mathrm{~min}$ [28]. After centrifugation, each extract was passed through a membrane filter $(0.22 \mathrm{~mm})$. The extraction and measurement of all samples were followed by the protocol described using a UV-Visible Spectrophotometer (UV2600, Shimadzu). The aqueous phase was used to determine the absorbance at $530 \mathrm{~nm}$ and $657 \mathrm{~nm}$. The total anthocyanin contents were quantified via the following equation: $\mathrm{Q}_{\text {Anthocyanins }}=\left(\mathrm{A}_{530}-0.25 \times \mathrm{A}_{657}\right) \times \mathrm{M}^{-1}$, where $\mathrm{Q}_{\text {Anthocyanins }}$ is the amount of anthocyanins, $\mathrm{A}_{530}$ and $\mathrm{A}_{657}$ are the absorptions at the indicated wavelengths, and $\mathrm{M}$ is the weight of the plant material in grams used for extraction.

\section{RESULTS}

\subsection{Cloning of RrGT1 and Sequence Analysis}

In the early stage, we screened out the $R r G T 1$ gene by comparing the differentially expressed genes in anthocyanin pathway in the $R$. rugosa transcriptome data of our laboratory. We obtained the $R r G T 1$ gene 3' terminal sequence of $277 \mathrm{bp}$ length by using nested PCR method (Figure 2(a)). The full-length sequence (Figure 2(b)) of the $R r G T 1$ gene was cloned by full-length primers (Table 1) and confirmed by sequencing. The $R r G T 1$ gene has a complete open reading frame from the starting codon ATG to the termination codon TAA, encodes a 386-amino acids protein and has a polyA tail (Figure 3 ). Sequence alignments with the NCBI database showed the $R r G T 1$ protein is a member of the GTB superfamily and has a typical plant secondary product glycosyltransferases conserved domain called PSPG consisting of 44 amino acid residues at the C-terminal.

\subsection{Temporal and Spatial Expression Patterns of the RrGT1 Gene}

The expression levels of the RrGT1 gene in $R$. rugosa "Zizhi", which significantly differed, were assessed during five flowering stages (Figure $4(a)$ ). The highest expression level was observed during the full opening stage, and the lowest was observed during the budding stage.

The expression levels of the RrGT1 gene in $R$. rugosa "Zizhi", which also significantly differed, were assessed in seven different tissue types (Figure 4(b)). The expression level in the leaves, stems and flower buds was relatively high but was relatively low in the other tissues. 
Table 1. Primers used in the present study.

\begin{tabular}{lll}
\hline \multicolumn{1}{c}{ Primer Name } & \multicolumn{1}{c}{ Sequence(5'-3') } & \multicolumn{1}{c}{ Description } \\
\hline 3' $R r G T 1$-F-outer & ATGTCTTGGTCCTCGCATTTC & 3'RACE for $R r G T 1$ \\
3' $R r G T 1$-F-inner & GAGGGGAAGAAGATGAGAGGTAAAG & \\
B26 & GACTCGAGTCGACATCGATTTTTTTTTTTTTTT & Full-length cDNA for $R r G T 1$ \\
$R r G T 1-\mathrm{F}$ & ATGTCAGGAAATCCACTGGATGC & \\
$R r G T 1-\mathrm{R}$ & TTAAGGCGAAGAAATCAAATCTACC & Generation for \\
p- $R r G T 1-\mathrm{F}$ & ACTAGTATGTCAGGAAATCCACTGGATGC & pCAMBIA1304- $r G T 1$ \\
p- $R r G T 1-\mathrm{R}$ & GGTGACCTTAAGGCGAAGAAATCAAATCTACC & \\
CpR-F & ATGGTAGATCTGACTAGTATGTCAGG & Confirmation for \\
CpR-R & CGAGCTGGTGACCTTAAGGC & pCAMBIA1304- $R r G T 1$ \\
$R r G A P D H-\mathrm{F}$ & TTCTGCCTGCTCTCAATG & \\
$R r G A P D H-\mathrm{R}$ & TGCCTTCTTCTCAAGTCTG & qRT-PCR for $R r G A P D H$ \\
q $R r G T 1-\mathrm{F}$ & GTATTTGCCAACACACTGAGTAA & \\
q $R r G T 1-\mathrm{R}$ & CTGCAGTGGTAATGAGAGGGAG & qRT-PCR for $R r G T 1$ \\
$N t A C T-\mathrm{F}$ & AGGGTTTGCTGGAGATGATG & \\
$N t A C T-\mathrm{R}$ & CGGGTTAAGAGGTGCTTCAG & qRT-PCR for $N t A C T$
\end{tabular}

Restriction enzyme site are underlined.

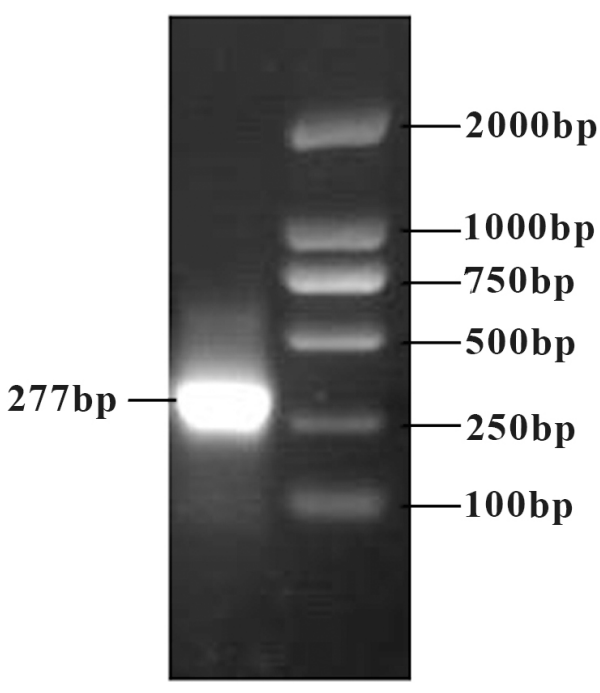

(a)

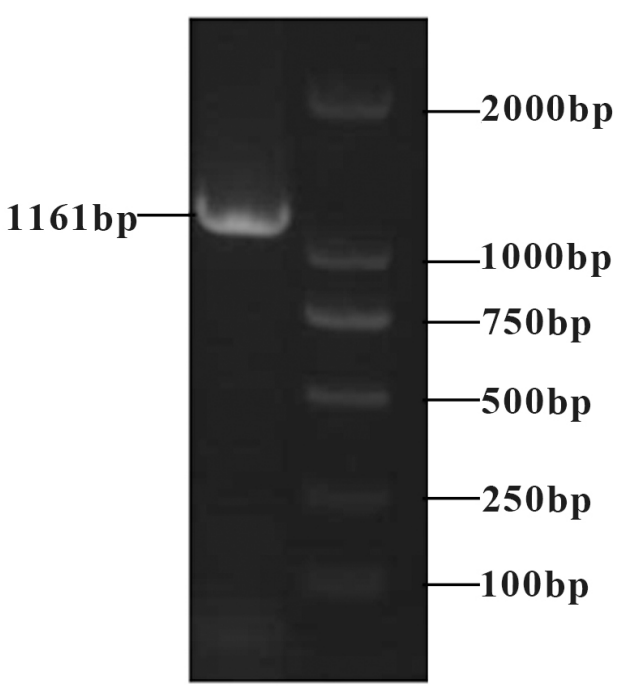

(b)

Figure 2. The results of 3'RACE amplification and full-length CDS amplification of the RrGT1 gene. (a) 3' RACE amplification product of the RrGT1 gene; (b) Full-length CDS amplification product of the RrGT1 gene.

\subsection{Construction and Verification of Recombinant Expression Vector pCAMBIA1304-RrGT1}

The empty vector and target gene were digested separately (Figure 5(a)) and linked together according to the pre-designed vector recombination technique route. In order to verify whether the recombination was successful, we designed a pair of specific primers (Table 1) to detect the transformation results by 

GCAGGTATAGACAAGGCCGTGGCGGAGAGAAGCGAGAGGCGGATCAGTTCCCTGATCACAGATGGATTCCTGGTC $\begin{array}{llllllllllllllllllllllllll}A & G & I & D & K & A & V & A & E & R & S & E & R & R & I & S & S & L & I & T & D & G & F & L & V\end{array}$ TTTGGTGGAGAGATTGCTGAGAGTTTGGGCATTCCATGGTTTCCTGTTTGGTTGCCCTTGGCCTACACCCTATCC $\begin{array}{llllllllllllllllllllllllll} & \text { F } & G & G & \text { E } & \text { I } & \text { A } & \text { E } & \text { S } & \text { L } & G & \text { I } & \text { P } & \text { W } & \text { F } & \text { P } & \text { V } & \text { W } & \text { L } & \text { P } & \text { L } & \text { A } & \text { Y } & \text { T } & \text { L } & S\end{array}$ GCTCATATATATACAGATTTGATTCGCCAGATCTGTGCCGAAGAAGCCATTGCAAACGGTGGTAATGAAGATTGG $\begin{array}{llllllllllllllllllllllllll}A & H & I & Y & T & D & \text { L } & I & R & Q & I & C & A & E & E & A & I & A & N & G & G & N & E & D & \text { W }\end{array}$ CGTCAGCACAAAAGGCTGGAAGTCATTCCAGGATTGTCTGCCATGCTCATTGCAGACGTGCCCGATGAAGTACTT $\begin{array}{lllllllllllllllllllllllll}R & Q & H & K & R & \text { L } & \text { E } & \text { V } & \text { I } & \text { P } & G & \text { L } & \text { S } & \text { A } & \text { M } & \text { L } & \text { I } & \text { A } & \text { D } & \text { V } & \text { P } & \text { D } & \text { E } & \text { V } & \text { L }\end{array}$ CCTAGCGAGTCGGTATTTGCCAACACACTGAGTAAACTCGGGCATGTGCTTCTGCAGCCGGCAGCCGTTGTCATG $\begin{array}{llllllllllllllllllllllllll}P & S & E & S & V & F & A & N & T & L & S & K & L & G & H & V & L & L & Q & P & A & A & V & V & M\end{array}$ AACTCGTACGAAGAACTAAGTTCCACAGCTCTCAACAATGATCTGAAATCCAAATTTAGGAAGGTGCTTAATGTA $\begin{array}{llllllllllllllllllllllllll}N & S & Y & E & E & \text { L } & S & S & T & A & L & N & N & D & L & K & S & K & F & R & K & V & L & N & V\end{array}$ GGGTTTCTCTCCCTCTCATTACCACTGCAGCCGCTTCCATCGTCAAACTCCGATGCCAGCGGCTGTCTTACGTGG $\begin{array}{llllllllllllllllllllllllll}G & F & L & S & L & S & L & P & L & Q & P & L & P & S & S & N & S & D & A & S & G & C & L & T & W\end{array}$ TTGGATGAGCAAGAAGCATCATCGTCTGTGGCGTATATTAGTTTCGGAACTGTGGCAGCCCCACCTCCGCACGAG $\begin{array}{llllllllllllllllllllllllll}\text { L } & D & \text { E } & Q & \text { E } & \text { A } & \text { S } & \text { S } & \text { S } & \text { V } & \text { A } & \text { Y } & \text { I } & \text { S } & \text { F } & G & \text { T } & \text { V } & \text { A } & \text { A } & \text { P } & \text { P } & \text { P } & \text { H } & \text { E }\end{array}$ TTGATTGCTTTGGCTGAGGCATTGGAAGAGAGTGGAATGCCATTTCTTTGGTCTCTTCGTGACAACATAAAGCAG $\begin{array}{llllllllllllllllllllllllll}\text { L } & I & A & L & A & E & A & L & E & E & S & G & M & P & F & L & \text { W } & S & L & R & D & N & I & K & Q\end{array}$ CTATTGCCGGATGGATTCGTCGAAAGGACTAACAAGCAGGGTAAAATAGTGGCATGGGCACCCCAAACTCAAGTT \begin{tabular}{llllllllllllllllllllllllll} 
L & $L$ & $P$ & $D$ & $G$ & $F$ & $V$ & $E$ & $R$ & $T$ & $N$ & $K$ & $Q$ & $G$ & $K$ & $I$ & $V$ & $A$ & W & $A$ & $P$ & $Q$ & $T$ & $Q$ & $V$ \\
\hline
\end{tabular} CTGGGACATGCTTCAATCGGGTTGTTTGTAACACACTGCGGGTGCAATTCGGTGTATGAAAGTATTGCAAATGGT \begin{tabular}{lllllllllllllllllllllllll} 
L & $G$ & $H$ & $A$ & $S$ & $I$ & $G$ & L & F & V & T & H & C & G & C & N & S & V & Y & E & S & I & A & N & $G$ \\
\hline
\end{tabular} GTGCCGATGATCTGCAGGCCGTTTTTCGGAGATCACCGAATGACGGGGCGGTTGATAGAGGAAGTATGGGGTGTT $\begin{array}{lllllllllllllllllllllllll}\mathrm{V} & \mathrm{P} & \mathrm{M} & \mathrm{I} & \mathrm{C} & \mathrm{R} & \mathrm{P} & \mathrm{F} & \mathrm{F} & \mathrm{G} & \mathrm{D} & \mathrm{H} & \mathrm{R} & \mathrm{M} & \mathrm{T} & \mathrm{G} & \mathrm{R} & \mathrm{L} & \mathrm{I} & \mathrm{E} & \mathrm{E} & \mathrm{V} & \mathrm{W} & \mathrm{G} & \mathrm{V}\end{array}$ GGAGTGAGCATTGAGGGTGGAGTATTTACGAGAAGTGGAGTACTCAAGAGCTTTGAGCTCATTTTGGCAAATGAA $\begin{array}{llllllllllllllllllllllllll}G & V & S & I & E & G & G & V & F & T & R & S & G & \text { V } & \text { L } & \text { K } & \text { S } & \text { F } & \text { E } & \text { L } & \text { I } & \text { L } & \text { A } & \text { N } & \text { E }\end{array}$ 1276 GAGGGGAAGAAGATGAGAGGTAAAGCACGAATGCTCAAAGAGGTTCTAGCAGAGGCTGCTGGGCCTTCCGGAAGT $\begin{array}{lllllllllllllllllllllllll}\text { E } & G & \text { K } & \text { K } & M & \text { R } & G & \text { K } & \text { A } & \text { R } & M & \text { L } & \text { K } & \text { E } & \text { V } & \text { L } & \text { A } & \text { E } & \text { A } & \text { A } & \text { G } & \text { P } & \text { S } & G & S\end{array}$ 1351 GCTGTGGAAGATTTTAGGACTTTGGTAGATTTGATTTCTTCGCCTTAAGCAACTAGGTTGTTGTTTTCTTTAATT $\begin{array}{lllllllllllllllll}\text { A } & \text { V } & \text { E } & \text { D } & \text { F } & R & \text { T } & \text { L } & \text { V } & \text { D } & \text { L } & \text { I } & \text { S } & \text { S } & \text { P } & * & *\end{array}$

1426 ATCGATCTGTTTTTGTCTGCCTTAATTGCAGTCTTACATCGTCTGCGGTCTTAATCGTATATTGTTTAAAAGTCT

1501 CTTCTTATTTAATAAAATATCTGACTGAAGACAAAAAAAAAAAAAAAAAAAA

Figure 3. cDNA sequence of $\operatorname{RrGT1}$ and its deduced amino acids. The red box shows the start codon and the termination codon and the amino acids they encode. The PSPG domains were underlined by the blue line.
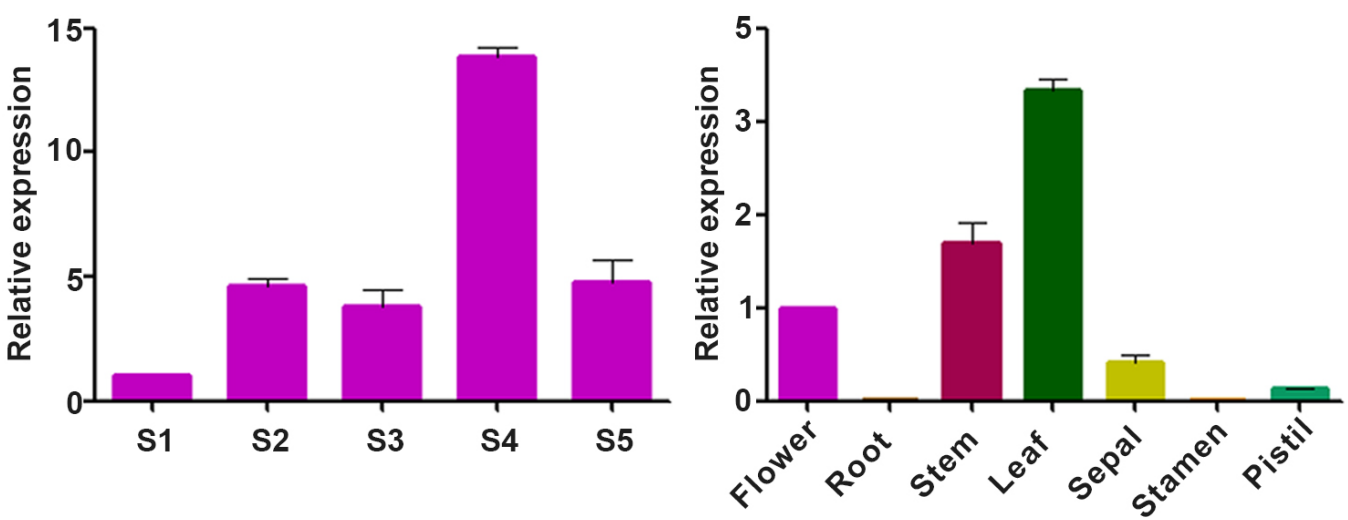

(a)

(b)

Figure 4. The temporal and spatial expression patterns of $R r G T 1$ in $R$. rugosa "Zizhi". The relative expressions of the $R r G T 1$ gene in five flowering stages (a) and seven different tissues (b). 


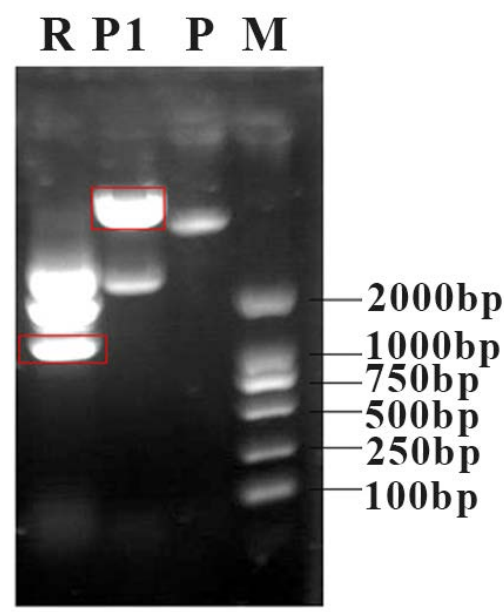

(a)

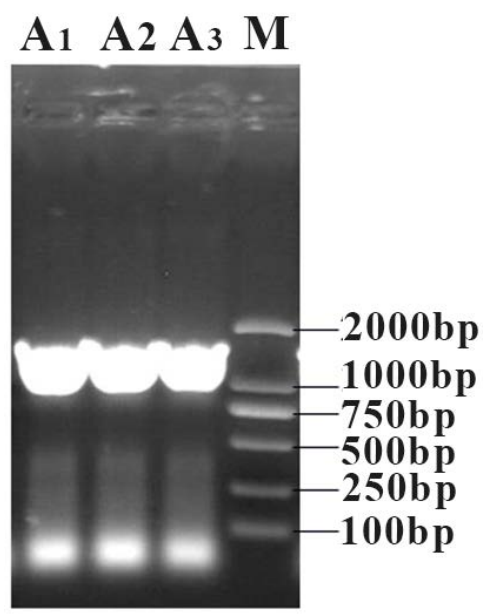

(b)

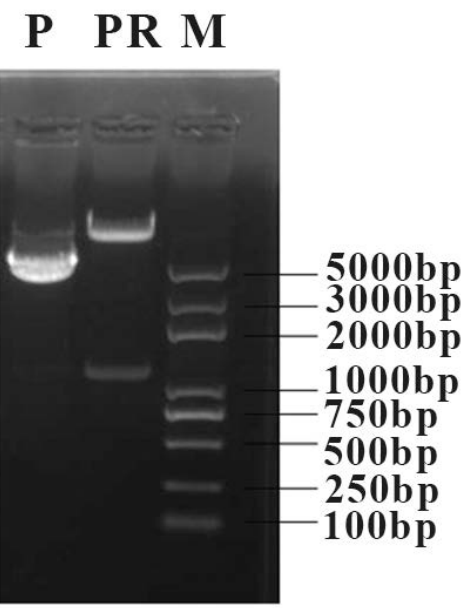

(c)

Figure 5. Construction and verification of recombinant expression vector pCAMBIA1304-RrGT1. (a) The vector and target gene were digested separately. M: Marker; P: The plasmid of the empty vector pCAMBIA1304 was used as the control; P1: The empty vector was digested by double enzymes; R: The plasmid carrying the target gene was digested by two enzymes. The red box indicates the portion that needs to be retained for subsequent operations; (b) The result of PCR verification. Three repeated PCR assays were performed on the recombinant vector and the results showed positive. M: Marker; A1, A2, A3 was the three repetitions; (c) Double enzyme digestion was used to verify the plasmid of the recombinant vector. M: Marker; $P$ : The plasmid of the empty vector pCAMBIA1304 was used as the control; PR: The plasmid of the recombinant vector was digested into two correct parts.

PCR, and the results showed positive (Figure 5(b)). In order to eliminate the possibility of false positivity and to further verify whether the target gene had been mutated or deleted during the recombination, we identified the target gene by double enzyme digestion and sequencing. The results of double enzyme digestion (Figure 5(c)) showed that the recombinant vector was cut into two distinct fragments: one part of the original vector and the other part of the target gene. The plasmid of the empty vector was used as the control. The sequencing results also showed that there was no base mutation and deletion in the target gene fragment of the embedded vector. The results above showed that the recombinant expression vector pCAMBIA1304- $R r G T 1$ was constructed successfully.

\subsection{Overexpression of the RrGT1 Gene Enhanced Anthocyanin Accumulation in Tobacco}

The $R r G T 1$ gene was ectopically expressed in tobacco using the binary vector pCAMBIA1304- $R r G T 1$. Six independent transgenic tobacco lines that overexpressed the $R r G T 1$ gene were obtained from Hyg-resistance selection and were cultured under the same conditions. PCR analysis confirmed the presence of the transformed $R r G T 1$ gene in all transgenic lines, and the absence of endogenous $R r G T 1$ in control group (wild-type) and empty vector group (plant was transformed with empty pCAMBIA1304 vector) tobacco plants. After RT-PCR (Figure 6(a)) and qRT-PCR (Figure 6(b)) tests, we excluded three false-positive lines, and the three identified transgenic lines, T1, T4, and T6, were used for further experiments.

Interestingly, there is no substantial difference in morphology between the transgenic plants and wild-type. However, transgenic tobacco lines harboring $R r G T 1$ were affected in flower color, with a remarkable deepening of petal pigmentation compared with the control group and empty vector group tobacco 


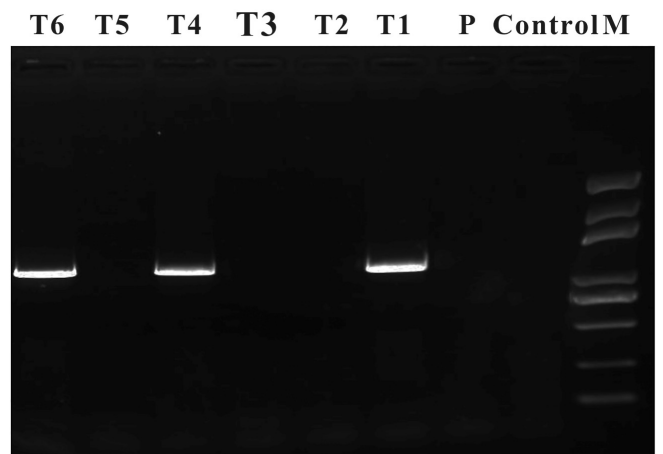

(a)

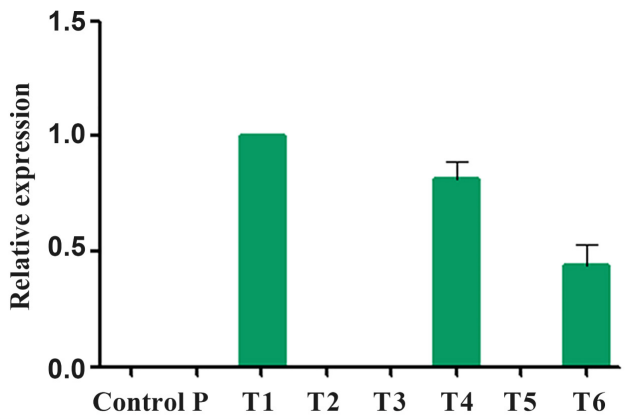

(b)

\section{$\begin{array}{lllll}\text { Control } & P & \text { T1 } & \text { T4 } & \text { T6 }\end{array}$}

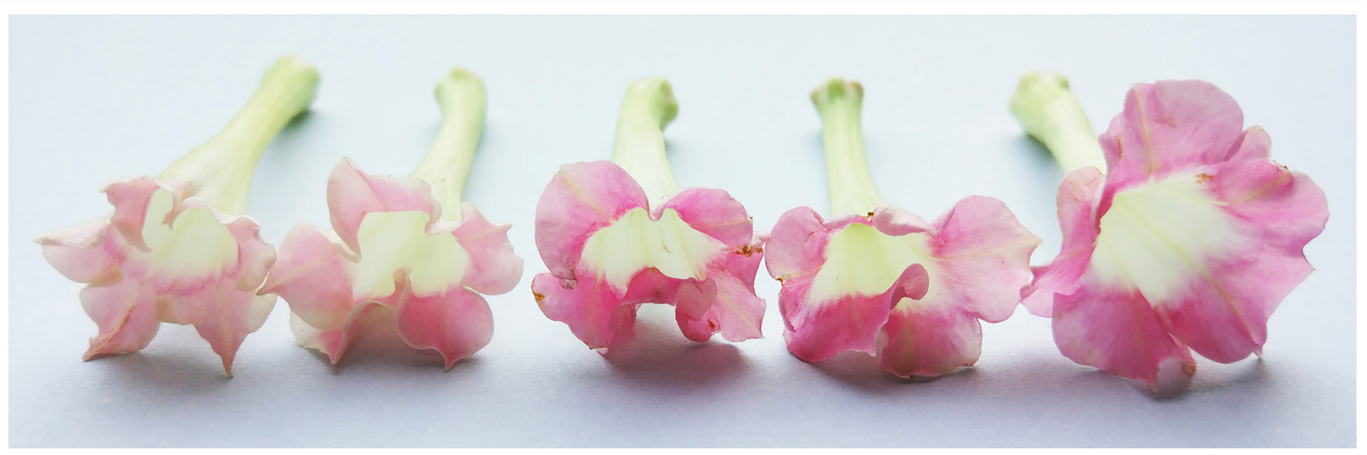

(c)

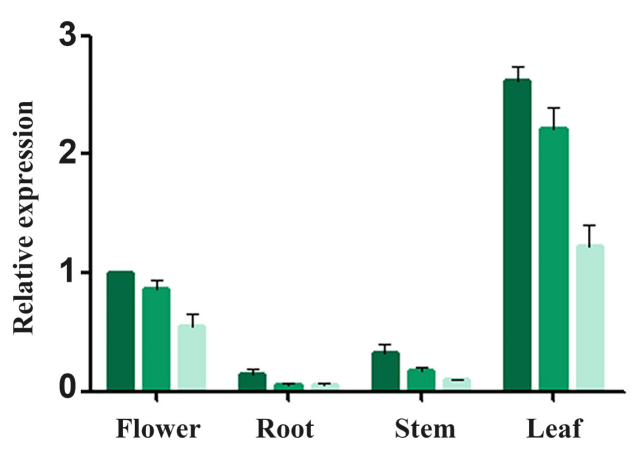

(d)

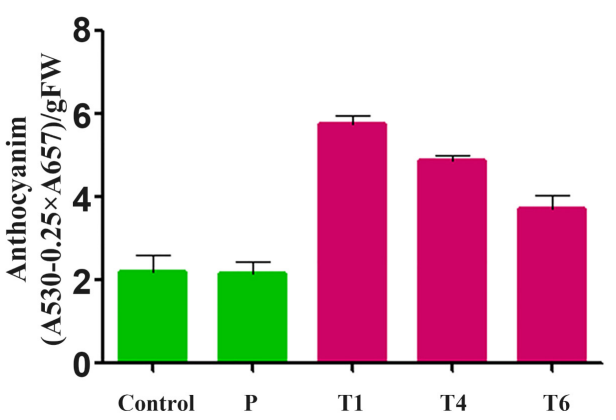

(e)

Figure 6. Molecular analyses of the $R r G T 1$-overexpressing tobacco plants. (a) PCR analysis of transgenic plants. M: Marker; Control, control group; $\mathrm{P}$, empty vector group; T1-T6, six resistant tobacco lines selected by tissue culture; (b) qRT-PCR analysis of transgenic plants; (c) Comparison of flower color phenotypes of transgenic tobacco; (d) The expression patterns of RrGT1 in transgenic tobacco; (e) Comparison of total anthocyanin content in transgenic tobacco flowers.

plants (Figure 6(c)). This change in the corolla color of transgenic tobacco was already visible prior to anthesis. To confirm that the deeper flower color was attributed from an increased pigment levels synthesized from the anthocyanin pathway, the total anthocyanins were determined. In the transgenic tobacco lines T1, T4, and T6, anthocyanin contents were improved to 2.58-, 2.03-, and 1.67-fold, respectively, as 
compared to the control group and empty vector group tobacco plants (Figure 6(e)). Notably, the expression of $R r G T 1$ in transgenic tobacco correlated with the pigmentation enhancement observed in the petals.

\subsection{The Expression Patterns of RrGT1 in Transgenic Tobacco}

The expression patterns of the $R r G T 1$ gene in three transgenic tobacco lines were analyzed by qRT-PCR (Figure 6(d)). The expression levels of $R r G T 1$, which significantly differed, were assessed in four different tissue types. The expression level in the leaves and flowers was relatively high but was relatively low in the stems and roots. Of all the tissue types of the three transgenic lines, the highest gene expression was T1, followed by T4, and the lowest was T6.

\section{DISCUSSION}

Although many genes have been reported to regulate the formation of flower color, there are few reports of downstream structural genes such as GTs. The final formation of anthocyanins depends on the glycosylation of GTs, so it is very important to elucidate the function and influence of the $R r G T 1$ gene in $R$. rugosa color formation. In this study, we successfully cloned $R r G T 1$ gene with full length cDNA of 1161 bp, encoding 386 amino acids from the petals of $R$. rugosa "Zizhi".

The expression levels of the $R r G T 1$ gene during flower development and in different tissues were investigated. It was found that the expression of the $R r G T 1$ gene showed a different trend during different flowering periods in $R$. rugosa "Zizhi", indicating that the expression of the $R r G T 1$ gene was developmentally regulated in the process of anthocyanin biosynthesis. Studies have shown that the accumulation of anthocyanin in red skinned sand pear, strawberries and litchi is positively correlated with the activity of UF3GT. Boss et al. [29] also detected the expression of UF3GT in the peels of red grape which accumulated anthocyanin, but not in other tissues of red grape and white grape without anthocyanin accumulation. Gong et al.'s [30] studies showed that the partial structural genes of Perilla frutescens anthocyanin metabolic pathway were only expressed in the leaves of red varieties, but not in green varieties or the expression in the green leaves was very low. About the tissue-specific expression in R. rugosa "Zizhi", besides the high expression level in flowers, it is worth mentioning that the stems of $R$. rugosa "Zizhi" are purple, which is consistent with the high expression level of the $R r G T 1$ gene. In addition, $R r G T 1$ was also highly expressed in the leaves, so we infer that $\operatorname{RrGT1}$ is also involved in the glycosylation of secondary metabolites in leaves and plays an important role.

To investigate the function of the $R r G T 1$ gene in anthocyanin biosynthesis in vivo, RrGT1 was first transferred into tobacco, which resulted in flower coloration enhancement of transgenic tobacco plants. It was found that the anthocyanins contents in the flowers of the three transgenic tobacco lines increased in varying degrees after the determination of the total anthocyanins contents. Therefore, it can be preliminarily confirmed that this result was caused by the overexpression of the $\operatorname{RrGT1}$ gene. And it also indicated that $R r G T 1$ could encode a functional glycosyltransferase (GT) protein for anthocyanin biosynthesis. In addition, after analyzing the tissue-specific expression of various transgenic tobacco lines, we found that the $R r G T 1$ gene was highly expressed not only in flowers but also in leaves. This phenomenon was consistent with the results of tissue specific expression analysis in the $R$. rugosa "Zizhi". Therefore, we speculated that the $\operatorname{RrGT1}$ gene may play an important role in the regulation of the growth and development of leaves even the whole plants in addition to the anthocyanin biosynthesis pathway. So, the results of the transgenic experiment proved that the exogenous $\operatorname{RrGT1}$ enzymes can also affect the synthesis of anthocyanins in different species. In other words, the function of $R r G T 1$ in anthocyanin biosynthesis and other aspects can be exchanged among different plant species.

In conclusion, we cloned the the $R r G T 1$ gene from $R$. rugosa "Zizhi" and proved by overexpression in tobacco that it could affect anthocyanin accumulation and thus the flower color. We believed that this research was beneficial to analyzing the molecular synthesis and regulation mechanism of anthocyanins, and also provided some important informations for the improvement of $R$. rugosa flower color in the future. 


\section{FUND}

This project was supported by the Agricultural Seed Project of Shandong Province ([2014] No. 96).

\section{CONFLICTS OF INTEREST}

The authors declare that the research was conducted in the absence of any commercial or financial relationships that could be construed as a potential conflict of interest.

\section{REFERENCES}

1. Li, M. (2006) Survey and Quality Evaluation of Shandong Rose Varieties. Shandong University of Traditional Chinese Medicine, Jinan.

2. Feng, L.G., Shao, D.W., Sheng, L.X., et al. (2009) Study on Investigation and Morphological Variation of Wild Rosa rugosa in China. Journal of Shandong Agricultural University (Natural Science Edition), 40, 484-488.

3. Chen, S.M., Zhu, X.R., Chen, F.D., et al. (2010) Expression Characteristics of Anthocyanin Structural Genes in Different Flower Color Chrysanthemum Cultivars. Journal of Northwest Plants, 3, 453-458.

4. Aizza, L.C. and Domelas, M.C. (2011) A Genomic Approach to Study Anthocyanin Synthesis and Flower Pigmentation in Passion Flowers. Journal of Nucleic Acids, 5, 371517.

5. Mehdy, M.C. and Lamb, C.J. (1988) Chalcone Isomerase cDNA Cloning and mRNA Induction by Fungal Elicitor, Wounding and Infection. Plant Physiology, 86, 182-186. https://doi.org/10.1104/pp.86.1.182

6. Liu, J., Feng, Q.F. and Zhang, J. (2005) Dihydroflavonol 4-Reductase Gene (DFR) and Flower Color Modulation. Plant Physiology Communications, 41, 715-719.

7. Springob, K., Nakajima, J.I., Yamazaki, M., et al. (2003) Recent Advances in the Biosynthesis and Accumutation of Anthocyanins. Natural Product Reports, 20, 288-303. https://doi.org/10.1039/b109542k

8. Rosati, C., Cadic, A., Duron, M., et al. (1999) Molecular Characterization of the Anthocyanidin Synthase Gene in Forsythia $\times$ Intermedia Reveals Organ-Specific Expression during Flower Development. Plant Science, 149, 73-79. https://doi.org/10.1016/S0168-9452(99)00146-6

9. Guo, F.D., Wang, X.Z., Wang, X.J., et al. (2011) Metabolic Regulation of Plants Anthocyanin. Chinese Bulletin of Life Sciences, 23, 938-944.

10. Ludwig, S.R., Habera, L.F., Dellaporta, S.L., et al. (1989) $L c$, a Member of the Maize $R$ Gene Family Responsible for Tissue-Specific Anthocyanin Production, Encodes a Protein Similar to Transcriptional Activators and Contains the myc-Homology Region. Proceedings of the National Academy of Sciences of the USA, 86, 7092-7096. https://doi.org/10.1073/pnas.86.18.7092

11. Carey, C.C., Strahle, J.T., Selinger, D.A., et al. (2004) Mutations in the Pale Aleurone Color1 Regulatory Gene of the Zea mays Anthocyanin Pathway Have Distinct Phenotypes Relative to the Functionally Similar Transparent Testa GLABRA1 Gene in Arabidopsis thaliana. The Plant Cell, 16, 450-464. https://doi.org/10.1105/tpc.018796

12. Grotewold, E. (2006) The Genetics and Biochemistry of Floral Pigments. Annual Review of Plant Biology, 57, 761-780. https://doi.org/10.1146/annurev.arplant.57.032905.105248

13. Allan, A.C., Hellens, R.P. and Laing, W.A. (2008) MYB Transcription Factors That Colour Our Fruit. Trends in Plant Science, 13, 99-102. https://doi.org/10.1016/j.tplants.2007.11.012

14. Kim, S.H., Lee, J.R., Hong, S.T., et al. (2003) Molecular Cloning and Analysis of Anthocyanin Biosynthesis Genes Preferentially Expressed in Apple skin. Plant Science, 165, 403-413. https://doi.org/10.1016/S0168-9452(03)00201-2

15. Martens, S., Preuß, A. and Matern, U. (2010) Multifunctional Flavonoid Dioxygenases: Flavonol and Anthocyanin Biosynthesis in Arabidopsis thaliana L. Phytochemistry, 71, 1040-1049. 
https://doi.org/10.1016/j.phytochem.2010.04.016

16. Ge, C.L., Huang, C.H. and Xu, X.B. (2012) Research on Anthocyanins Biosynthesis in Fruit. Acta Horticulturae Sinica, 39, 1655-1664.

17. Forkmann, G. (2010) Flavonoids as Flower Pigments: The Formation of the Natural Spectrum and Its Extension by Genetic Engineering. Plant Breeding, 106, 1-26. https://doi.org/10.1111/j.1439-0523.1991.tb00474.x

18. Yonekura-Sakakibara, K., Nakayama, T., Yamazaki, M., et al. (2009) Modification and Stabilization of Anthocyanins. Springer, New York, 169-190.

19. Zheng, Z.L. (1994) Flower Color Gene Engineering of Flower Crops. Northern Horticulture, 3, 37-38.

20. Vogt, T. and Jones, P. (2000) Glycosyltransferases in Plant Natural Product Synthesis: Characterization of a Supergene Family. Trends in Plant Science, 5, 380-386. https://doi.org/10.1016/S1360-1385(00)01720-9

21. Tohge, T., Nishiyama, Y., Hirai, M.Y., et al. (2005) Functional Genomics by Integrated Analysis of Metabolome and Transcriptome of Arabidopsis Plants Over-Expressing an MYB Transcription Factor. The Plant Journal, 42, 218-235. https://doi.org/10.1111/j.1365-313X.2005.02371.x

22. Goto, T., Kondo, T., Tamura, H., et al. (1982) Structure of Gentiodelphin, an Acylated Anthocyanin Isolated from Gentiana makinoi, That Is Stable in Dilute Aqueous Solution. Tetrahedron Letters, 23, 3695-3698. https://doi.org/10.1016/S0040-4039(00)88660-8

23. Hall, D., Yuan, X.X., Murata, J., et al. (2012) Molecular Cloning and Biochemical Characterization of the UDP-Glucose: Flavonoid 3-0-Glucosyltransferase from Concord Grape (Vitis labrusca). Phytochemistry, 74, 90-99. https://doi.org/10.1016/j.phytochem.2011.10.007

24. Tanaka, Y., Yoshikazu, K., Fukuchi-Mlizutani, M., et al. (1996) Molecular and Biochemical Characterization of Three Anthocyanin Synthetic Enzymes from Gentiana triflora. Plant and Cell Physiology, 37, 711-716. https://doi.org/10.1093/oxfordjournals.pcp.a029004

25. Yamazaki, M., Yamagishi, E., Gong, Z.Z., et al. (2002) Two Flavonoid Glucosyltransferases from Petunia hybrida: Molecular Cloning, Biochemical Properties and Developmentally Regulated Expression. Plant Molecular Biology, 48, 401-411. https://doi.org/10.1023/A:1014043214943

26. Horsch, R., Fry, J.E., Hoffmann, N.L., et al. (1985) A Simple and General Method for Transferring Genes into Plants. Science, 227, 1229-1232. https://doi.org/10.1126/science.227.4691.1229

27. Schmittgen, T.D. and Livak, K.J. (2008) Analyzing Real-Time PCR Data by the Comparative C(T) Method. Nature Protocols, 3, 1101. https://doi.org/10.1038/nprot.2008.73

28. Yang, Q., Yuan, T. and Sun, X.B. (2015) Preliminary Studies on the Changes of Flower Color during the Flowering Period in Two Tree Peony Cultivars. Acta Horticulturae Sinica, 42, 930-938.

29. Boss, P.K., Davies, C. and Robinson, S.P. (1996) Expression of Anthocyanin Biosynthesis Pathway Genes in Red and White Grapes. Plant Molecular Biology, 32, 565-569. https://doi.org/10.1007/BF00019111

30. Gong, Z.Z., Yamazaki, M., Sugiyama, M., et al. (1997) Cloning and Molecular Analysis of Structural Genes Involved in Anthocyanin Biosynthesis and Expressed in a Forma-Specific Manner in Perilla frutescens. Plant Molecular Biology, 35, 915-927. https://doi.org/10.1023/A:1005959203396 


\section{ABBREVIATIONS}

cDNA, complementary DNA;

RACE, rapid amplification of cDNA ends;

CDS, coding DNA sequence;

$\mathrm{PCR}$, polymerase chain reaction;

qRT-PCR, quantitative real-time polymerase chain reaction;

HPLC, high-performance liquid chromatography. 